\title{
Iatrogenic Oesophageal Perforation in an Extremely Low Birth Weight Newhorn
}

JCR Marie Janaillac', Ramy El-Jalbout ${ }^{2}$, Anie Lapointe', Christian Lachance', Gabriel Altit' Division of 'Neonatology and '2Radiology, CHU Sainte-Justine, Université de Montreal, Montreal, Canada.

\section{Abstract:}

We report a rare case of an extremely low birth weight (ELBW) baby with oesophageal perforation secondary to an oro-gastric tube insertion and concomitant milk pleural effusion diagnosed on chest radiography. Area of perforation was determined with lateral chest radiography. This is the first case described in the literature of a milk pleural effusion in the context of perforation of the inferior third of the oesophagus in an ELBW infant.

Key words: Abdomen, Birth Weight, Esophageal Perforation, Milk, Pleural Effusion, Pregnancy.

\section{Introduction}

Traumatic oesophageal perforation, as an iatrogenic complication in the ELBW population, has been described since 1961 [1]. Subsequently, it has remained of rare occurrence, although the incidence is unknown. latrogenic etiologies include multiple unsuccessful endotracheal intubations and installation of a feeding tube or a suction catheter. The occurrence decreases with technical skills but the risk remains high in the ELBW babies. The most common site for iatrogenic neonatal oesophageal perforation is in the upper oesophagus. Here we report a rare case of an ELBW baby with oesophageal perforation secondary to an orogastric tube insertion.

\section{Case Report}

A baby boy was delivered at $25+5$ weeks of gestational age by caesarean section for abnormal foetal heart rate variability. He was severely growth restricted with abnormal in-utero Doppler. Birth weight was 430 grams. Patient was intubated due to increasing respiratory distress and umbilical arterial and venous lines were inserted. Initial chest and abdominal radiographies were normal. The first oro-gastric tube was inserted at 14 hours of life to begin trophic feeds with breast milk. Feedings were reinstituted at 39 hours of life with a new oro-gastric tube after a brief pause due to abdominal distension. Minutes later, acute respiratory deterioration occurred and oxygen requirements increased from $\mathrm{FiO}_{2} 30 \%$ to $70 \%$. Chest radiography was done and the gastric tube was found to be in the superior right abdomen, below the liver margin, with an associated right-sided pleural effusion and pneumo-mediastinum [Fig.1]. Lateral chest radiography confirmed the distal

\section{Corresponding Author: Dr. Marie Janaillac}

Email: mariejanaillac@hotmail.com

Received: March 19, 2016 | Accepted: June 16, 2016 | Published Online: August 15, 2016

This is an Open Access article distributed under the terms of the Creative Commons Attribution License (creativecommons.org/licenses/by/3.0)

Conflict of interest: None declared | Source of funding: Nil | DOl: http://dx.doi.org/10.17659/01.2016.0092 
third oesophageal perforation with the tube in the posterior mediastinum [Fig.2]. Surgery consultation was done, the tube was immediately removed and antibiotics were started. Pleural effusion was confirmed by ultrasound and was quickly drained due to hemodynamic and respiratory instability. Patient's condition worsened in the following hours with refractory hypotension, anuria and severe metabolic acidosis despite inotropic support that lead to the patient's death at 49 hours of life. Blood cultures were positive for Lactobacillus paracasei. Parents declined autopsy. After internal review, the appropriate oro-gastric tubes were inserted, without difficulty, by skilled nurses who followed the routine procedure.

\section{Discussion}

This case report is about the rare occurrence of oesophageal perforation with a milk pleural effusion in an ELBW infant diagnosed on chest radiography. Pleural effusion with milk has been described in the literature in the context of oesophageal obstruction, in pharyngeal or oesophageal pseudo-diverticulum [2], in the context of a contained cervical perforation and in pneumothoraxes resulting from puncture into the thoracic cavity [2-4]. This is the first report of milk effusion and lactobacillus sepsis secondary to oesophageal perforation.

Typical location of traumatic oesophageal perforation is at the pharyngo-oesophageal junction. This area is more susceptible to injury being the narrowest point of the oesophagus and with a tendency for reflex muscular constriction upon instrumentation. Furthermore, cervical vertebrae compress the posterior oesophageal wall and hyperextension of the neck during instrumentation further increases the risk of perforation [5]. The most common site for iatrogenic neonatal oesophageal perforation is in the upper oesophagus. Injury of the inferior third of the oesophagus has been rarely described and may occur due to a vertical false

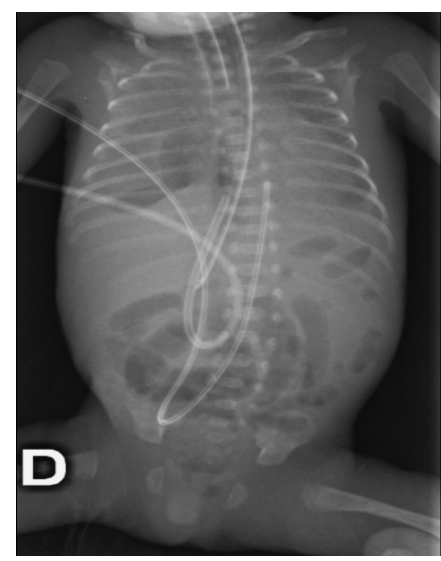

Fig.1: Chest and abdominal radiography done after acute respiratory deterioration. The orogastric tube is found in the right upper abdominal quadrant in a paravertebral location, with an associated right-sided pleural effusion and pneumomediastinum.

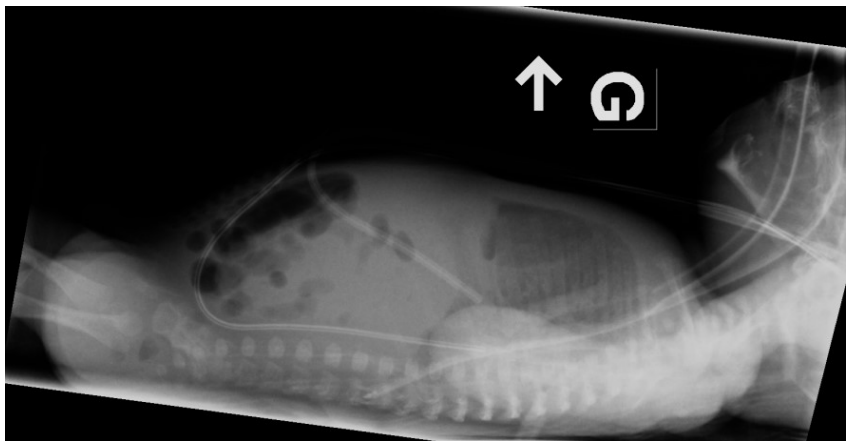

Fig.2: Lateral chest radiography showing the tip of the oro-gastric tube in the posterior mediastinum. Perforation occurred in the distal third of the oesophagus.

tract through the submucosal layer of the posterior oesophagus [6].

Traumatic injury should always be suspected with unexplained nasal or oro-pharyngeal bleeding. In several neonatal intensive care units, gastric tubes are replaced daily or at each feed increasing the risk of traumatic mucosal injury. When oesophageal 
perforation is suspected, antero-posterior and lateral chest radiography should be done $[2,3,7]$. Opacification may help to confirm perforation when radiography is not conclusive. However, this later is often difficult to perform in these critically ill premature infants. Management of iatrogenic oesophageal perforation is controversial. Immediate surgical care and conservative management have both been described. Most cases in the literature were treated with antibiotics and feeding interruption for a period of 10 to 20 days [8] but death incurred in $20 \%$ to $30 \%$ of them $[5,7]$.

Oesophageal perforation repair by endoscopy has been described by Soong et al. [6]. It allows an earlier diagnosis and permits proper gastric tube placement, bypassing the area of perforation with continuation of enteral feed. However, this technique is challenging in smaller infants and comes with an added risk of translocation secondary to air insufflation and instrumentation.

\section{Conclusions}

The iatrogenic oesophageal perforation secondary to an oro-gastric tube was diagnosed on chest radiography by the combined presence of a malpositioned nasogastric tube and an ipsilateral pleural effusion confirmed on chest ultrasound. Oesophageal perforation is a serious complication associated with high mortality in the ELBW population. A high index of suspicion and early radiological diagnosis should help clinicians improve the medical and surgical management of this complication.

\section{References}

1. Warden HD, Mucha SJ. Esophageal perforation due to trauma in the newborn. A case report. Archives of Surgery. 1961;83:813-815.

2. Shah PS, Dunn MS, Shah VS. Oesophageal perforation in preterm neonates: not an innocent bystander. Journal of Paediatrics and Child Health. 2003;39(9):697-699.

3. Jones KE, Wagener S, Willetts IE, Lakhoo K. Oesophageal perforation in extreme prematurity. BMJ Case Reports. 2012;2012.

4. Suryawanshi P, Dahat A, Nagpal R, Malshe N, Kalrao $V$. A rare case of accidental esophageal perforation in an extremely low birth weight neonate. Journal of Clinical and Diagnostic Research. 2014;8(6):PD01-2.

5. Sapin E, Gumpert L, Bonnard A, Carricaburu $E$, Sava $E$, Contencin $P$, et al. latrogenic pharyngoesophageal perforation in premature infants. European journal of Pediatric Surgery. 2000;10(2):83-87.

6. Soong WJ. Endoscopic diagnosis and management of iatrogenic cervical esophageal perforation in extremely premature infants. Journal of the Chinese Medical Association. 2007;70(4):171-175.

7. Filippi L, Pezzati $M$, Poggi C. Use of polyvinyl feeding tubes and iatrogenic pharyngo-oesophageal perforation in verylow-birthweight infants. Acta Paediatrica. 2005;94(12):1825-1828.

8. Johnson DE, Foker J, Munson DP, Nelson A, Athinarayanan $\mathrm{P}$, Thompson TR. Management of esophageal and pharyngeal perforation in the newborn infant. Pediatrics. 1982;70(4):592596. 